\title{
Stability regions of fractional first order controllers applied to fractional order delay systems
}

\author{
Karim Saadaoui \\ Department of Computer Engineering, \\ College of Computers and Information Technology, \\ Taif University, Taif, Saudi Arabia
}

Received: February 26, 2021. Revised: April 12, 2021. Accepted: April 14, 2021. Published: April 22, 2021.

\begin{abstract}
This paper focuses on the problem of stabilizing fractional order time delay systems by fractional first order controllers. A solution is proposed to find the set of all stability regions in the controller's parameter space. The D-decomposition method is employed to find the real root boundary and complex root boundaries which are used to identify the stability regions. Illustrative examples are given to show the effectiveness of the proposed approach, and it is remarked that the stability region obtained for the fractional order controller is larger than the non-fractional controller.
\end{abstract}

Keywords - Fractional order control, first order controller, stability, time delay systems.

\section{INTRODUCTION}

$\mathrm{C}$ ONTROL of fractional order systems has gained the attention of many researchers in the last decade. In fact, many real-life processes in electrochemistry, material science and biology are better represented by fractional order systems. These systems are characterized by differential equations of arbitrary order, not necessarily integer order. Fractional calculus is a solution to better represent these systems by incorporating non-integer order slopes in their frequency responses and hence dealing with poles and zeros of fractional power. See [1] and the references therein for examples of fractional order systems.

In this paper, we study stability of fractional order delay systems. Actually, many industrial applications inherently contain time delay which is generated by physical phenomenon such as information transport, time lags between interconnected systems and many other factors. Combining fractional order and time delay makes stability analysis and controller design more difficult and challenging [2]. In fact, time delay systems possess an infinite number of roots which makes straightforward stability check almost impossible.

Determining all the stabilizing controllers for a given linear time invariant systems is an important step in designing optimal controllers, as they represent the search space for satisfying further design criteria. The problem becomes more difficult by fixing the order and structure of the controllers used [3]-[4]. On the other hand, most controllers used in industry are fixed structure, low order controllers being first order, proportional $(\mathrm{P})$, proportional derivative $(\mathrm{PD})$, proportional integral (PI), or proportional integral derivative (PID) controllers. Obtaining the set of all stabilizing controllers with fixed order and structure is an important task and has many advantages. For all the above reasons, there was an extensive literature on stabilizing time delay systems by low order controllers [5]-[11] such as first order controllers in [7], PI controllers in [8], and PID controllers [9].

In the above-mentioned papers, the stabilizing problem is solved for systems which are described by integer order differential equations and few studies dealt with fractional controllers applied to fractional order systems with time delay [10]. It is known that using fractional order controllers can improve the overall performance of the closed loop system of time delay systems and can also improve robustness [2]. In fact, fractional order controllers outperform integer order controllers in many situations [2]. Many papers in the literature dealt with tuning of fractional order PID controllers, and little attention was paid to studying fractional order lead lag controllers, although first order lead lag controllers are also widely used in industry. In this paper, we determine 
the set of all stabilizing fractional first order controllers for fractional order time delay systems.

The paper is structured as follows. The second section gives some preliminary results. Section III describes our approach for determining stabilizing fractional first order controllers for fractional order time delay systems. In section IV, illustrative examples are given. Finally, the last section presents some concluding remarks.

\section{PRELIMINARIES}

In this paper, the objective is to determine all the stabilizing parameters $\left(\alpha_{1}, \alpha_{2}, \alpha_{3}\right)$ of a fractional first order controller applied to the feedback system shown in Fig. 1.

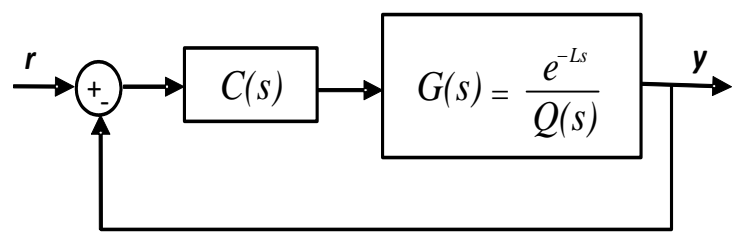

Fig. 1 Fractional first order control structure The fractional first order controller can be written as $C(s)=\frac{\alpha_{2} s^{\lambda_{2}}+\alpha_{3}}{s^{\lambda_{1}+\alpha_{1}}}$

where $\lambda_{1}$ and $\lambda_{2}$ are the fractional orders. The fractional first order controller can be considered as a generalization of the conventional first order controller as it involves a pole and a zero with non-integer slopes in their frequency responses. Theoretically, $\lambda_{1}$ and $\lambda_{2}$ can take any positive, real value. However, like the fractional PID controller case, the interval of the fractional orders is limited to $(0,2)$. For the case $\lambda_{1}=$ $\lambda_{2}=1$, we obtain the classical first order controller. $\alpha_{1}=0$ and $\lambda_{1}=\lambda_{2}$ give a fractional PI controller, and taking $\lambda_{1}=0$ results in a fractional PD controller.

The system to be stabilized is a fractional order time delay system with a transfer function given by

$G(s)=\frac{e^{-L s}}{Q(s)}$

where $L>0$ is the delay, and $Q(s)$ is a fractional polynomial where the powers of $s$ can take any positive real value, not necessarily an integer. The denominator $Q(s)$ can be written as

$$
\begin{aligned}
Q(s) & =b_{n} s^{\beta_{n}}+b_{n-1} s^{\beta_{n-1}}+\cdots+b_{1} s^{\beta_{1}}+b_{0} s^{\beta_{0}}, \\
& =\sum_{i=0}^{n} b_{i} s^{\beta_{i}},
\end{aligned}
$$

where $\beta_{n}>\beta_{n-1}>\cdots>\beta_{1}>\beta_{0} \geq 0$ are arbitrary real positive numbers.

The resulting closed-loop characteristic equation is

$\Delta^{*}\left(s, \alpha_{1}, \alpha_{2}, \alpha_{3}\right)=\left(s^{\lambda_{1}}+\alpha_{1}\right) Q(s)+\left(\alpha_{2} s^{\lambda_{2}}+\alpha_{3}\right) e^{-L s}$.

Using the expression of $Q(s)$ given in (3), we get the following fractional order characteristic equation $\Delta^{*}\left(s, \alpha_{1}, \alpha_{2}, \alpha_{3}\right)=\left(s^{\lambda_{1}}+\alpha_{1}\right)\left(b_{n} s^{\beta_{n}}+b_{n-1} s^{\beta_{n-1}}+\cdots+\right.$

$$
\left.b_{1} s^{\beta_{1}}+b_{0} s^{\beta_{0}}\right)+\left(\alpha_{2} s^{\lambda_{2}}+\alpha_{3}\right) e^{-L s} \text {. }
$$

The objective is to find all values of $\left(\alpha_{1}, \alpha_{2}, \alpha_{3}\right)$ for which the quasi-polynomial $\Delta^{*}\left(s, \alpha_{1}, \alpha_{2}, \alpha_{3}\right)$ has all its roots in the open left-half of the s-plane. We are searching a stability domain $S$, in the space of the controller's parameters $\left(\alpha_{1}, \alpha_{2}, \alpha_{3}\right)$, by employing the D-decomposition method [12]-[14]. The principle of this method is based on the fact that roots of the quasipolynomial (5) change continuously as the coefficients of the quasi-polynomial are changed continuously. As a result, a stable quasi-polynomial becomes unstable if and only if at least one of its roots moves from the left half plane to the right half plane by crossing the imaginary axis. Using this fact, the D-decomposition method partitions the controller's parameter's space into regions such that each region has a fixed number of roots in the left half complex plane. Therefore, stability can be inferred by selecting a point inside the region and using any stability classical checking methods, such as Nyquist criterion.

\section{DETERMINING STABILITY REGIONS OF FRACTIONAL FIRST ORDER CONTROLLERS}

In this section, the stabilizing regions in the parameter space of the controller are obtained by applying the Ddecomposition method. The boundaries of the stability domains are determined by replacing $s$ by $j \omega$ in the fractional order characteristic equation (5), and equating the real and imaginary parts to zero, and sweeping over values of $\omega \geq 0$. Real root boundary and complex root boundaries are calculated. Note that the term $e^{L S}$ has no finite roots, consequently the quasi-polynomial $\Delta^{*}(s)$ and $\Delta(s)=\Delta^{*}(s) e^{L S}$ have the same roots, therefore stability of $\Delta(s)$ is equivalent to stability of $\Delta^{*}(s)$. In the sequel, the quasi-polynomial $\Delta(s)$ will be used to infer stability of the closed-loop system of Fig. 1, where $\Delta(s)$ is given by

$$
\begin{aligned}
\Delta(s) & =\left(s^{\lambda_{1}}+\alpha_{1}\right) Q(s) e^{L s}+\left(\alpha_{2} s^{\lambda_{2}}+\alpha_{3}\right) \\
& =\left(s^{\lambda_{1}}+\alpha_{1}\right)\left(\sum_{i=0}^{n} b_{i} s^{\beta_{i}}\right) e^{L s}+\left(\alpha_{2} s^{\lambda_{2}}+\alpha_{3}\right) .
\end{aligned}
$$

Assuming that $s^{\beta_{0}}=1$ in (1), the real root boundary is found by setting $\omega=0$ in $\Delta\left(j \omega, \alpha_{1}, \alpha_{2}, \alpha_{3}\right)$, which results in an equation of straight line expressed as $\alpha_{3}=-b_{0} \alpha_{1}$. 
The complex root boundaries can be determined by substituting $s=j \omega$ in (6), equating the real an imaginary parts of $\Delta(j \omega)$ to zero and solving for $\alpha_{2}$ and $\alpha_{3}$ in terms of $\alpha_{1} . \Delta(s)$ can be written as

$\Delta(s)=Q_{0}(s)+\alpha_{1} Q_{1}(s)+\alpha_{2} Q_{2}(s)+\alpha_{3}$, where

$Q_{0}(s)=\left(b_{n} s^{\beta_{n}+\lambda_{1}}+\cdots+b_{1} s^{\beta_{1}+\lambda_{1}}+b_{0} s^{\beta_{0}+\lambda_{1}}\right) e^{L s}$,

$Q_{1}(s)=\left(b_{n} s^{\beta_{n}}+b_{n-1} s^{\beta_{n-1}}+\cdots+b_{1} s^{\beta_{1}}+b_{0} s^{\beta_{0}}\right) e^{L s}$,

$Q_{2}(s)=s^{\lambda_{2}}$.

Replacing $s$ by $j \omega$, we obtain

$\Delta(j \omega)=Q_{0}(j \omega)+\alpha_{1} Q_{1}(j \omega)+\alpha_{2} Q_{1}(j \omega)+\alpha_{3}$.

Using (12), we have to deal with fractional order powers of $j$, which can be written as

$$
\begin{aligned}
j^{\beta_{i}+\lambda_{1}} & =\cos \left(\left(\beta_{i}+\lambda_{1}\right) \frac{\pi}{2}\right)+j \sin \left(\left(\beta_{i}+\lambda_{1}\right) \frac{\pi}{2}\right), \\
& =x_{i}+j y_{i}, \\
j^{\beta_{i}}= & \cos \left(\beta_{i} \frac{\pi}{2}\right)+j \sin \left(\beta_{i} \frac{\pi}{2}\right), \\
= & z_{i}+j t_{i},
\end{aligned}
$$

for $i=1, \ldots, n$. Hence, $\Delta(j \omega)$ can be expressed as

$$
\begin{aligned}
\Delta(j \omega)= & \sum_{i=0}^{n} b_{i} \omega^{\beta_{i}+\lambda_{1}}\left(x_{i}+j y_{i}\right)(\cos (L w)+j \sin (L w)) \\
& +\alpha_{1} \sum_{i=0}^{n} b_{i} \omega^{\beta_{i}}\left(z_{i}+j t_{i}\right)(\cos (L w)+j \sin (L w)) \\
& +\left(\alpha_{2} \omega^{\lambda_{2}}\left(\cos \left(\lambda_{2} \frac{\pi}{2}\right)+j \sin \left(\lambda_{2} \frac{\pi}{2}\right)\right)+\alpha_{3}\right) .
\end{aligned}
$$

Separating the above equation into real and imaginary parts, we obtain

$$
\begin{aligned}
\Delta(j \omega)= & \left(Q_{0 r}(j \omega)+j Q_{0 j}(j \omega)\right)+\alpha_{1}\left(Q_{1 r}(j \omega)+j Q_{1 j}(j \omega)\right) \\
& +\alpha_{2}\left(Q_{2 r}(j \omega)+j Q_{2 j}(j \omega)\right)+\alpha_{3} .
\end{aligned}
$$

Let $\mathfrak{R}\left(\omega, \alpha_{1}, \alpha_{2}, \alpha_{3}\right)$ and $\square\left(\omega, \alpha_{1}, \alpha_{2}\right)$ denote the real and imaginary parts of $\Delta(j \omega)$, respectively. Then, (16) can be written as

$\Delta(j \omega)=\square\left(\omega, \alpha_{1}, \alpha_{2}, \alpha_{3}\right)+j \square\left(\omega, \alpha_{1}, \alpha_{2}\right)$,

(17)

where

$\square\left(\omega, \alpha_{1}, \alpha_{2}, \alpha_{3}\right)=Q_{0 r}(j \omega)+\alpha_{1} Q_{1 r}(j \omega)+\alpha_{2} Q_{2 r}(j \omega)+\alpha_{3}$, and

$\square\left(\omega, \alpha_{1}, \alpha_{2}\right)=Q_{0 j}(j \omega)+\alpha_{1} Q_{1 j}(j \omega)+\alpha_{2} Q_{2 j}(j \omega)$.

Equating the real and imaginary parts of (17) to zero, we get

$Q_{0 r}(j \omega)+\alpha_{1} Q_{1 r}(j \omega)+\alpha_{2} Q_{2 r}(j \omega)+\alpha_{3}=0$,

$Q_{0 j}(j \omega)+\alpha_{1} Q_{1 j}(j \omega)+\alpha_{2} Q_{2 j}(j \omega)=0$,

\section{Where}

$Q_{0 r}(\omega)=\sum_{i=0}^{n} b_{i} \omega^{\beta_{i}+\lambda_{1}}\left(x_{i} \cos (L \omega)-y_{i} \sin (L \omega)\right)$,

$Q_{1 r}(\omega)=\sum_{i=0}^{n} b_{i} \omega^{\beta_{i}}\left(z_{i} \cos (L \omega)-t_{i} \sin (L \omega)\right)$,

$Q_{2 r}(j \omega)=\omega^{\lambda_{2}} \cos \left(\lambda_{2} \frac{\pi}{2}\right)$

$Q_{0 j}(\omega)=\sum_{i=0}^{n} b_{i} \omega^{\beta_{i}+\lambda_{1}}\left(y_{i} \cos (L \omega)+x_{i} \sin (L \omega)\right)$

$Q_{1 j}(\omega)=\sum_{i=0}^{n} b_{i} \omega^{\beta_{i}}\left(t_{i} \cos (L \omega)+z_{i} \sin (L \omega)\right)$,

$Q_{2 j}(j \omega)=\omega^{\lambda_{2}} \sin \left(\lambda_{2} \frac{\pi}{2}\right)$.

Solving the system (18) and (19), $\alpha_{2}$ and $\alpha_{3}$ are obtained in terms of $\alpha_{1}$ as

$\alpha_{2}=-\frac{Q_{0 j}(\omega)+\alpha_{1} Q_{1 j}(\omega)}{Q_{2 j}(\omega)}$

$\alpha_{3}=-\left(Q_{0 r}(\omega)+\alpha_{1} Q_{1 r}(\omega)+\alpha_{2} Q_{2 r}(\omega)\right)$

for a fixed value of $\alpha_{1}$, equations (26) and (27) together with equation (7) partition the complex plane into rootinvariant regions, among which the stability region, if any, can be determined.

\section{ILLUSTRATIVE EXAMPLES}

In this section, two illustrative examples are given to show the application of the procedure described in the previous section.

Example 1: Stabilizing fractional first order controllers are determined for the fractional order time delay system studied in [15], having the following transfer function

$$
G(s)=\frac{e^{-0.5 s}}{s^{1.5}} \text {. }
$$

Using (6) the fractional order characteristic equation of the control system is given by

$\Delta(s)=\left(s^{1.5+\lambda_{1}}+\alpha_{1} s^{1.5}\right) e^{0.5 s}+\left(\alpha_{2} s^{\lambda_{2}}+\alpha_{3}\right)$,
$=Q_{0}(s)+\alpha_{1} Q_{1}(s)+\alpha_{2} Q_{2}(s)+\alpha_{3}$,

with

$Q_{0}(s)=s^{1.5+\lambda_{1}} e^{0.5 s}$,

$Q_{1}(s)=s^{1.5} e^{0.5 s}$,

$Q_{2}(s)=s^{\lambda_{2}}$.

Fixing $\alpha_{1}$ and using (26) and (27) to determine $\alpha_{2}$ and $\alpha_{3}$, we obtain

$\alpha_{2}=-\frac{1}{\omega^{\lambda_{2}} \sin \left(\lambda_{2} \frac{\pi}{2}\right)}\left[\omega^{1.5+\lambda_{1}}\left(\cos \left(\left(1.5+\lambda_{1}\right) \frac{\pi}{2}\right) \sin (0.5 \omega)+\right.\right.$ $\left.\sin \left(\left(1.5+\lambda_{1}\right) \frac{\pi}{2}\right) \cos (0.5 \omega)\right)+\alpha_{1} \omega^{1.5}(\cos (3 \pi / 4)) \sin (0.5 \omega)+$ $\sin (3 \pi / 4)) \cos (0.5 \omega))]$,

$\alpha_{3}=\omega^{1.5+\lambda_{1}}\left[\sin \left(\left(1.5+\lambda_{1}\right) \frac{\pi}{2}\right) \cos (0.5 \omega)-\cos ((1.5+\right.$

$\left.\left.\left.\lambda_{1}\right) \frac{\pi}{2}\right) \cos (0.5 \omega)\right]+\alpha_{1} \omega^{1.5}[(\sin (3 \pi / 4)) \sin (0.5 \omega)-$ $\cos (3 \pi / 4)) \cos (0.5 \omega))]-\alpha_{2} \omega^{\lambda_{2}} \cos \left(\lambda_{2} \frac{\pi}{2}\right)$.

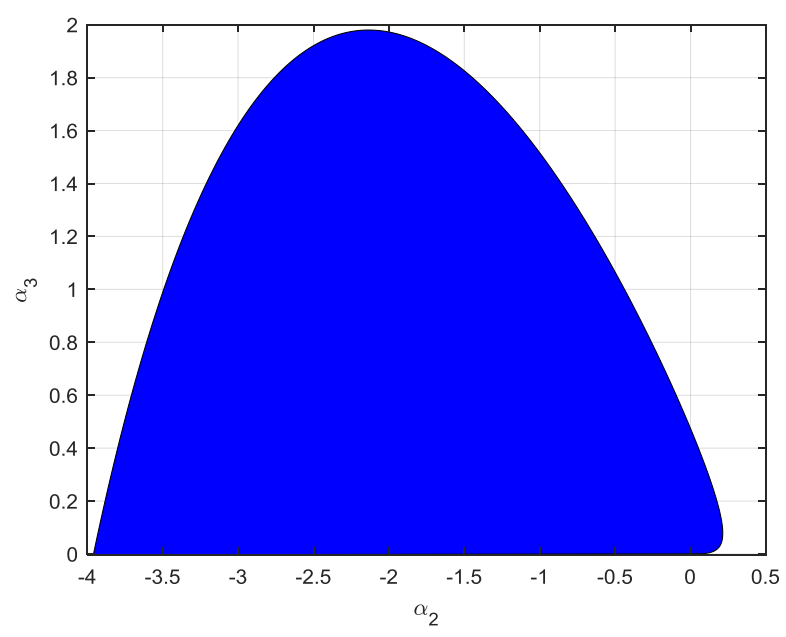

Fig. 2 Stability region in $\left(\alpha_{2}, \alpha_{3}\right)$ plane with $\alpha_{1}=1, \lambda_{1}=$ 1 and $\lambda_{2}=1$ 
Using (7), the real root boundary is a straight line given by $\alpha_{3}=0$. First, let us fix the value of $\alpha_{1}=1$ and choose $\lambda_{1}=1$ and $\lambda_{2}=1$, this corresponds to the case of a non-fractional first order controller. Equations (26) and (27) are used to get the complex root boundary curve as shown in Fig. 2. The shaded region represents the stability region in the $\left(\alpha_{2}, \alpha_{3}\right)$ plane. Repeating the same steps above with $\lambda_{1}=0.5$ and $\lambda_{2}=0.5$, a larger stability region is obtained as shown in Fig. 3. This clearly shows the advantage of using a fractional order controller in terms of robustness and non-fragility.

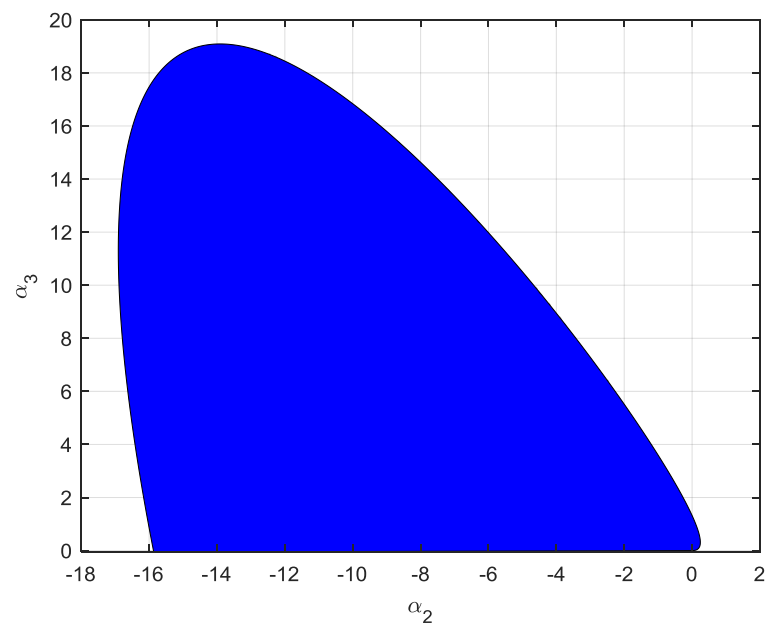

Fig. 3 Stability region in $\left(\alpha_{2}, \alpha_{3}\right)$ plane with $\alpha_{1}=1, \lambda_{1}=$ 0.5 and $\lambda_{2}=0.5$

In Fig. 4 the stability regions are obtained as a 3D plot by choosing $\alpha_{1}=1, \lambda_{1}=0.5$ and varying $\lambda_{2}$ between 0 and 2. It is noticed that as $\lambda_{2}$ approaches zero, the stability region becomes larger. Fig. 5 shows the stability regions when $\alpha_{1}=1, \lambda_{2}=0.5$ and $\lambda_{1} \in(0,2)$.

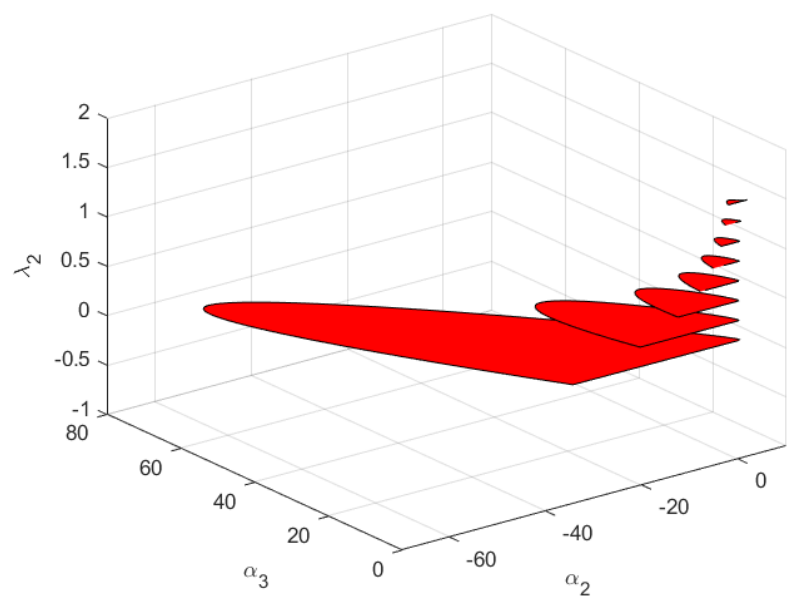

Fig. 4 Stability regions with $\alpha_{1}=1, \lambda_{1}=0.5$ and $\lambda_{2} \in(0,2)$

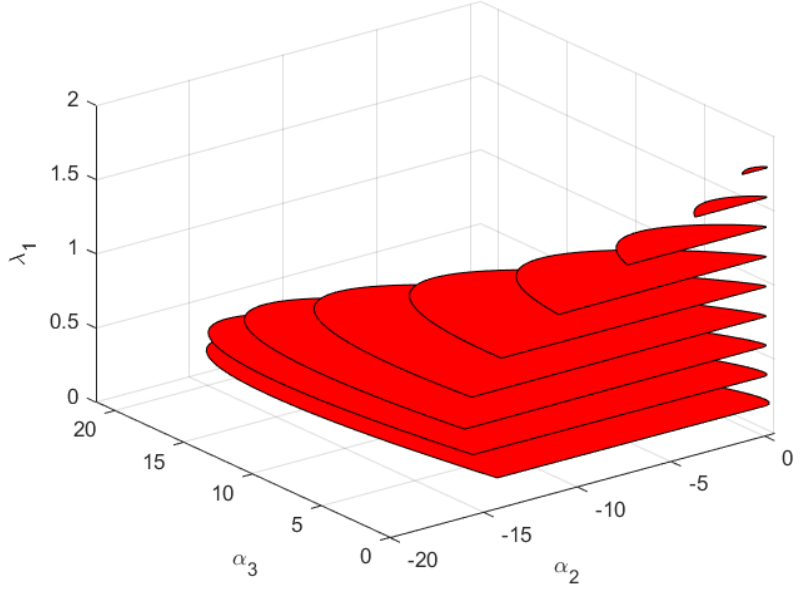

Fig. 5 Stability regions with $\alpha_{1}=1, \lambda_{2}=0.5$ and $\lambda_{1} \in(0,2)$

Example 2: In this example, we consider stabilizing the fractional order delay system given by

$G(s)=\frac{e^{-0.2 s}}{s^{1.2}+3 s^{0.7}}$

by fractional first order controllers. In order to see the effect of the fractional order $\lambda_{2}$ on the stability regions, the following parameters were chosen: $\alpha_{1}=0$ and $\lambda_{1}=$ 1. Fig. 6 shows the stability regions in the $\left(\alpha_{2}, \alpha_{3}\right)$ plane for different values of $\lambda_{2}$.

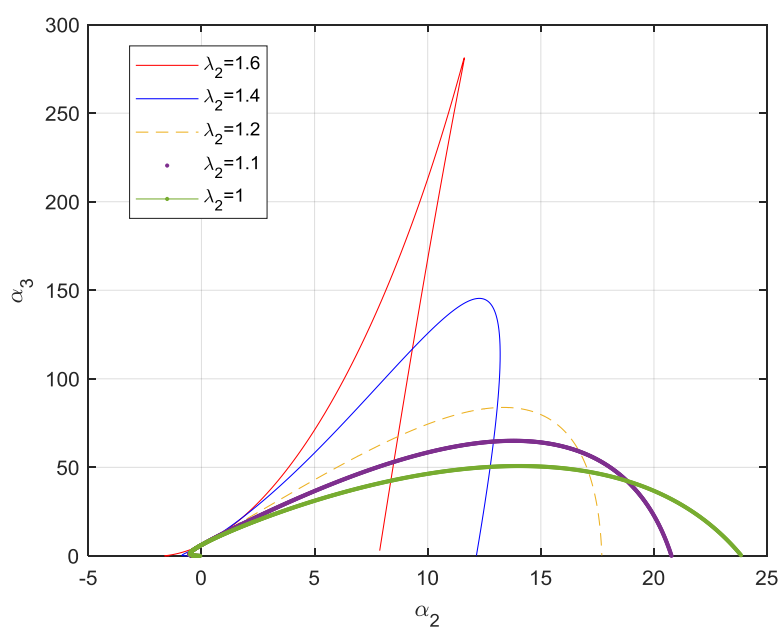

Fig. 6 Stability regions with $\alpha_{1}=0$ and $\lambda_{1}=1$

\section{CONCLUSION}

In this article, a stabilization method is proposed to obtain stabilizing fractional first order controllers applied to a fractional order time delay system. The given approach is based on using the $\mathrm{D}$-decomposition method to determine the stability domain boundaries via the real root boundary and complex root boundaries. The 
method can be used to design controllers with specified gain and phase margins. It is well known that stability is an essential step in any controller design, hence the proposed approach is useful as a first step towards designing optimal fractional controllers for fractional order time delay systems. This line of research will be investigated in future work.

\section{REFERENCES}

[1] C. A. Monje, Y. Q. Chen, B. M. Vinagre, D. Xue, and V. Feliu, Fractional order systems and controls. Fundamentals and applications, Springer, 2010.

[2] I. Birs, C. Muresan, I. Nascu, and C. Inoescu, "A survey of recent advances in fractional order control for time delay systems," IEEE Access, vol. 7, pp. 30951-30965, 2019.

[3] K. Saadaoui and A. B. Ozguler, "Stablizing first-order controllers with desired stability region," Control and Intelligent Systems, vol. 37, pp. 31-38, 2009.

[4] N. Tan, I. Kaya, C. Yeroglu and D. P. Atherton, "Computation of stabilizing PI and PID controllers using the stabilizing boundary locus," Energy Conversion \& Management, vol. 47, pp. 3045-3058, 2006.

[5] S. Testouri, K. Saadaoui, and M. Benrejeb, "Analytical design of first order controllers for the TCP/AQM systems with time delay," International Journal of Information Technology Control and Automation, vol. 2, pp. 27-37, 2012.

[6] S. Testouri, K. Saadaoui, and M. Benrejeb, "Stabilizing first-order controllers for TCP/AQM networks," Computer Technology and Application, vol. 2, pp. 979984, 2011.

[7] G. O. Young, H. Alihani, and A. Madady, "First-order controller design for second order integrating systems with time delay," in Proceedings of IEEE Conference on Control Applications, Hyderabad, 2013.

[8] S. Elmadssia, K. Saadaoui, and M., Benrejeb, "PI controller design for time delay systems using an extension of the Hermit-Biehler theorem," Journal of Industrial Engineering, vol. 13, pp. 1-6, 2013.

[9] K. Pandey, I. K. Andey, and L. Dewan, "Stabilizing sets of PID controllers for mini-mum phase integrating processes with dead time," in Proceedings of the 13th Int. Conference on Circuits, Systems, Electronics, Control \& Signal Processing, CSECS, Lisbon, 2014.

[10]A. B. Hmed, M. Amairi, and M. Aoun, "Robust stabilization and control using fractional order integrator," Trans. Inst. Meas. Control, vol. 39, pp. 1559-1576, 2017.

[11] K. Saadaoui, "On stabilizing fractional order time delay systems by first order controllers," International Journal of Systems Applications, Engineering \& Development, vol. 12, pp. 8-11, 2018.

[12] E. N. Gryazina, and B. T. Polyak, "Stability regions in the parameter space: D-decomposition revisited," Automatica, vol. 42, pp. 13-26, 2006.

[13] N. Hohenbicher, and J. Ackermann, "Computing stable regions in parameter spaces for a class of quasi- polynomials," in Proceedings of 4th IFAC Workshop on Time Delay Systems, TDS' 03, Rocquencourt,2003.

[14] J. Osuisky, and V. Vesely, "Modification of Neimark Dpartition method for desired phase margin," in Proceedings of the International Conference on Cybernetics and Informatics, Vyšná Boca, 2010.

[15]S. E. Hamamci, "An algorithm for stabilization of fractional order time delay systems using fractional order PID controllers," IEEE Transactions on Automatic Control, 52, 1964-1969, 2007.

\section{Creative Commons Attribution License 4.0 (Attribution 4.0 International, CC BY 4.0)}

This article is published under the terms of the Creative Commons Attribution License 4.0 https://creativecommons.org/licenses/by/4.0/deed.en US 